\title{
Polymerase chain reaction based human leucocyte antigen genotyping for the investigation of suspected gastrointestinal biopsy contamination
}

\author{
A C Bateman, S J Turner, J M Theaker, B F Warren, W M Howell
}

\begin{abstract}
Background-Mislabelling or contamination of surgical specimens may lead to diagnostic inaccuracy, particularly within gastrointestinal pathology when multiple small mucosal biopsy specimens are commonly taken, and where a tiny fragment of foreign tissue may be indistinguishable from true biopsy material using histological assessment alone.
\end{abstract}

Aims-To assess the utility of polymerase chain reaction (PCR) based human leucocyte antigen (HLA) genotyping techniques for the investigation of potentially mislabelled or contaminated gastrointestinal biopsy specimens.

Patients-Ten cases (28 samples) in which mislabelling or contamination was suspected, comprising four upper gastrointestinal tract biopsies and six colonoscopic biopsy series.

Methods-Direct and nested PCRsequence specific primer (SSP) based HLA class II genotyping was performed on DNA extracted from formalin fixed and paraffin wax embedded tissue (23 samples) or peripheral blood leucocytes (five samples).

Results-A full HLA-DRB1 genotype was determined in all 28 samples. In seven cases the HLA-DRB1 genotype of the putative contaminant was different to that of the corresponding reference tissue, confirming different individual origins for the contaminant and reference material. In one case the contaminant tissue was shown to possess the same HLA-DRB1 alleles as a second patient (probable source). In the remaining three cases the same HLA-DRB1 alleles were detected within the potential contaminant and reference tissues.

Conclusions-PCR based HLA class II genotyping is a valuable tool for investigating potential contamination or mislabelling within gastrointestinal biopsy specimens and this report has confirmed contamination in seven of ten cases studied.

(Gut 1999;45:259-263)

Correspondence to:

Dr A C Bateman,

Department of

Histopathology, Level E,

South Block, Southampton

General Hospital, Tremona

Road, Southampton

SO16 6YD, UK

Accepted for publication 17 February 1999

Contamination or mislabelling of surgical pathology specimens can potentially lead to serious errors in diagnosis. Specimen contamination may pose a particular problem in gastrointestinal pathology because many specimens comprise multiple tiny mucosal biopsy samples and therefore a tiny tissue fragment that is "carried over" between specimens may closely resemble "true" biopsy fragments in size and nature. The individual origin of such contaminants is not likely to be ascertainable from histological assessment alone. Furthermore, many gastrointestinal biopsies are performed either when maligancy is clinically suspected or during screening for neoplasia, with such biopsies usually representing the gold standard for diagnosis. Therefore, specimen contamination or mislabelling could lead to assignment of a neoplastic condition to the incorrect patient, with potentially serious consequences for all involved.

We have assessed the utility of two previously described polymerase chain reaction (PCR) based human leucocyte antigen (HLA) class II genotyping methods ${ }^{12}$ for the investigation of ten potentially mislabelled or contaminated gastrointestinal biopsy cases. Both methods were developed within our laboratory (Histocompatibility and Immunogenetics, Southampton) and were initially derived from routine tissue typing techniques used for bone marrow and solid organ transplantation programmes and disease association studies. ${ }^{34}$ HLA class II genotyping was achieved in both techniques via a parallel series of PCRs, each of which contained a sequence specific primer (SSP) pair designed to produce a PCR product if a particular HLA class II allele or allele group was present (PCR-SSP genotyping). The nested PCR-SSP method incorporated an initial generic PCR amplification step in order to improve sensitivity significantly and to enable successful typing with very limited quantities of template DNA.

\section{Case reports}

CASE 1

A 66 year old woman underwent gastroscopy (distal oesophagitis but no obvious macroscopic lesion) and oesophageal biopsy during investigation for cervical lymph node and pulmonary metastatic carcinoma of unknown primary origin. Histology revealed one fragment of inflamed gastric type mucosa together with a fragment of severely dysplastic stratified

Abbreviations used in this paper: HLA, human leucocyte antigen; PCR, polymerase chain reaction; SSP, sequence specific primer. 
squamous epithelium, raising suspicion of contamination.

CASE 2

A 73 year old man underwent gastroscopy (no discrete macroscopic lesions) and two random biopsies for dyspepsia, one of which showed signet ring adenocarcinoma. Repeat gastroscopy confirmed no macroscopic evidence of tumour. Initial macroscopic and histological examination of the subsequent gastrectomy specimen revealed no evidence of tumour, raising the possibility that the initial biopsy was mislabelled or contaminated.

CASE 3

A 45 year old man underwent gastroscopy (duodenitis but no discrete lesions) for dyspepsia. Histology revealed gastritis together with a single tiny neoplastic epithelial fragment, raising suspicion of contamination.

CASE 4

A 70 year old woman underwent gastroscopy (oesophageal varices but no macroscopic tumour) and duodenal biopsy for mild anaemia. A fragment of normal duodenal mucosa was received along with a fragment of oesophageal tissue infiltrated by adenocarcinoma, strongly suggesting contamination. Six patients seen in the same clinic were clinically considered as potential origins for the malignant tissue.

CASE 5

A 20 year old woman with possible Crohn's disease underwent colonoscopy and multiple biopsies for diarrhoea and right iliac fossa pain. One biopsy contained an additional dysplastic tissue fragment, raising suspicion of contamination.

CASE 6

A 59 year old woman underwent colonoscopy (one small polyp, otherwise macroscopically normal) with multiple mucosal biopsies for change in bowel habit. The polyp was histologically confirmed as a tubular adenoma. A fragment of poorly differentiated carcinoma was identified among the remaining biopsy specimens and contamination was suspected.

CASE 7

A 35 year old man with suspected irritable bowel syndrome underwent colonoscopy (macroscopically normal) and multiple biopsies. All biopsy specimens comprised normal large bowel mucosa except one which consisted of dysplastic and villous colonic epithelium, raising suspicion of contamination.

CASE 8

A 77 year old man underwent colonoscopy (no discrete macroscopic lesions) with multiple biopsies for rectal bleeding. All biopsy specimens showed quiescent chronic inflammatory bowel disease apart from one additional fragment of neoplastic epithelium, raising suspicion of contamination.
CASE 9

A 28 year old woman underwent colonoscopy (no discrete macroscopic lesions) and multiple biopsies for ulcerative colitis. The transverse colon biopsy specimen comprised dysplastic colonic epithelium. A colonic adenoma from a second patient was laboratory processed on the same day, raising suspicion that the dysplastic epithelium within the first patient's biopsy had originated from the second patient.

\section{CASE 10}

A 56 year old woman underwent flexible sigmoidoscopy (no discrete macroscopic lesions) and biopsy during a colorectal cancer screening trial. Histology revealed normal colonic mucosa together with a tiny fragment of neoplastic epithelium, raising suspicion of contamination.

\section{Methods}

In each case, the HLA class II genotype of the neoplastic tissue was compared with the remaining (reference) tissue within the same biopsy specimen. In case 2, the genotype of the gastrectomy specimen was also assessed. In case 4, biopsy specimens from two additional patients were genotyped as well as peripheral blood samples from the index patient and four further patients. In case 9, the genotype of the second patient's biopsy was also assessed.

DNA EXTRACTION-PERIPHERAL BLOOD SAMPLES Where applicable (case 4), $1 \mathrm{ml}$ of EDTA (Merck) blood from each individual underwent red cell lysis (buffer: $0.32 \mathrm{M}$ sucrose (Sigma, Poole, UK), 1\% Triton X-100 (Sigma), 5 mM $\mathrm{MgCl}_{2}$ (Merck, Poole, UK), $12 \mathrm{mM}$ Tris- $\mathrm{HCl}$ $\mathrm{pH} 7.5$ (Sigma)) followed by 30 minute Proteinase K (Sigma) digestion, with a DNA yield of approximately $250 \mathrm{ng} / \mu \mathrm{l}$.

DNA EXTRACTION-FORMALIN FIXED AND

PARAFFIN WAX EMBEDDED TISSUE

As described previously, ${ }^{4}$ five $15 \mu \mathrm{m}$ sections were cut from each paraffin wax block under investigation, and dewaxed. In one case (case 5) the putative contaminant was only present within one $5 \mu \mathrm{m}$ haematoxylin and eosin stained paraffin wax section and was carefully scraped off the glass slide after cover slip removal. The resulting cellular material underwent overnight Proteinase $\mathrm{K}$ digestion. DNA concentration and integrity was estimated by running a $2 \mu \mathrm{l}$ aliquot on a $1 \%$ agarose gel (Sigma).

NESTED PCR-SSP HLA CLASS II GENOTYPING

This method has been developed and previously described by our laboratory. ${ }^{2}$ The second exons within HLA-DRB1, 3, 4, and 5 genes were amplified in the first round PCR and $10 \mu \mathrm{l}$ of reaction product was run on a $2 \%$ agarose gel to estimate DNA yield. This PCR product was used as the DNA template for the second round PCR-SSP step, generally at 1 in 10000 dilution. Where the DNA yield from the initial PCR was low, dilutions of up to 1 in 20 were used, the optimum dilution being determined empirically. The PCR-SSP step was performed 
Table 1 PCR HLA class II genotyping results

\begin{tabular}{|c|c|c|c|c|c|c|}
\hline Case & & Method & $H L A-D R B 1$ & $H L A-D R B 3,4,5$ & $H L A-D Q B 1$ & Conclusion \\
\hline \multirow[t]{2}{*}{1} & Dysplastic tissue & $\mathrm{nSSP}$ & 04,13 & $53(4) \dagger$ & NA & Same HLA type \\
\hline & Reference tissue & SSP & 04,13 & $53(4)$ & 06,08 & \\
\hline \multirow[t]{3}{*}{2} & Neoplastic tissue & nSSP & 0301,15 & $51(5), 52(3)$ & NA & Same HLA type \\
\hline & Gastritis tissue & $\mathrm{nSSP}$ & 0301,15 & $51(5), 52(3)$ & NA & \\
\hline & Gastrectomy tissue & SSP & 0301,15 & $51(5), 52(3)$ & 02,06 & \\
\hline \multirow[t]{2}{*}{3} & Neoplastic tissue & $\mathrm{nSSP}$ & 0301,14 & $52(3)$ & $\mathrm{NA}$ & Contaminant \\
\hline & Reference tissue & $\mathrm{nSSP}$ & 0301,04 & $52(3), 53(4)$ & NA & \\
\hline \multirow[t]{8}{*}{4} & Neoplastic tissue & $\mathrm{nSSP}$ & 0301,04 & $52(3), 53(4)$ & NA & Contaminant \\
\hline & Reference (pb) & SSP & 04,04 & $53(4)$ & 07,08 & \\
\hline & Second patient & $\mathrm{nSSP}$ & 04,10 & $53(4)$ & NA & \\
\hline & Third patient & $\mathrm{nSSP}$ & 0701,0701 & $53(4)$ & NA & \\
\hline & Fourth patient $(\mathrm{pb})$ & SSP & 12,12 & No association & $0301 / 4,0301 / 4$ & \\
\hline & Fifth patient $(\mathrm{pb})$ & SSP & 0301,13 & $52(3)$ & 02,06 & \\
\hline & Sixth patient $(\mathrm{pb})$ & SSP & 0701,1302 & $52(3), 53(4)$ & 02,06 & \\
\hline & Seventh patient $(\mathrm{pb})$ & SSP & 0301,1302 & $52(3)$ & 02,06 & \\
\hline \multirow[t]{2}{*}{5} & Dysplastic tissue & nSSP & 0103, 0301 & $52(3)$ & $\mathrm{NA}$ & Same HLA type \\
\hline & Reference tissue & $\mathrm{nSSP}$ & 0103,0301 & $52(3)$ & NA & \\
\hline \multirow[t]{2}{*}{6} & Malignant tissue & $\mathrm{nSSP}$ & 03,11 & $52(3)$ & NA & Contaminant \\
\hline & Reference tissue & SSP & 01,1402 & $52(3)$ & 05,07 & \\
\hline \multirow[t]{2}{*}{7} & Dysplastic tissue & $\mathrm{nSSP}$ & 01,04 & $53(4)$ & NA & Contaminant \\
\hline & Reference tissue & SSP & 12,1302 & $52(3)$ & 06,07 & \\
\hline \multirow[t]{2}{*}{8} & Dysplastic tissue & $\mathrm{nSSP}$ & 0301,13 & $52(3)$ & NA & Contaminant \\
\hline & Reference tissue & SSP & 0301,14 & $52(3)$ & $02 \ddagger$ & \\
\hline \multirow[t]{3}{*}{9} & Dysplastic tissue & $\mathrm{nSSP}$ & 01,15 & $51(5)$ & $\mathrm{NA}$ & Contaminant \\
\hline & Reference tissue & SSP & 15,15 & $51(5)$ & 06 & \\
\hline & Second patient & SSP & 01,15 & $51(5)$ & 07,08 & \\
\hline \multirow[t]{2}{*}{10} & Neoplastic tissue & $\mathrm{nSSP}$ & 0301,11 & $52(3)$ & NA & Contaminant \\
\hline & Remaining tissue & nSSP & 03,14 & $52(3)$ & NA & \\
\hline
\end{tabular}

SSP, direct PCR-SSP genotyping; nSSP, nested PCR-SSP genotyping; pb, peripheral blood derived DNA (all other results derived from formalin fixed, paraffin wax embedded DNA).

†The expected DRB3 (DR52) gene was not detected.

$¥$ The expected DQB $1^{\star} 05$ allele was not detected.

In cases 1,2 , and 5 , the chance of two unselected white Western individuals possessing the same HLA-DRB1 alleles is: case 1 $\left(\right.$ HLA-DRB $\left.{ }^{\star} 04,13\right) 6 \%$; case $2\left(\right.$ HLA-DRB $\left.{ }^{\star} 0301,15\right) 8 \%$; case $5\left(\right.$ HLA-DRB $\left.1{ }^{\star} 0103,0301\right)<1 \%{ }^{4}{ }^{11}$

using panels of primers specific to hypervariable sequence motifs within the second exon of the HLA-DRB1 gene, plus primers specific for the HLA-DRB3, 4, and 5 genes. All primer sequences were derived from the literature, ${ }^{6-8}$ or were designed in house, using published HLA-DRB1 allele sequences, ${ }^{9}$ and were combined into 23 mixes, detecting 19 HLA-DRB1 alleles (or allele groups) plus the presence of the HLA-DRB3, -DRB4, or -DRB5 genes. PCR products were visualised by running the entire reaction mix on a $2 \%$ agarose gel. The first round PCRs were prepared in a laminar flow hood and within a separate room from the second round PCRs in order to minimise the risk of DNA contamination.

DIRECT PCR-SSP HLA GENOTYPING (NO

PREAMPLIFICATION STEP)

This method was used where sufficient intact DNA was extractable from paraffin wax biopsy specimens (cases 1 and 6-9) and for peripheral blood derived DNA (case 4). In addition to the HLA-DRB1, 3, 4, and 5 loci, this technique was used to examine the HLA-DQB1 locus. As described previously by our laboratory, ${ }^{1}$ this method was similar to that used for the second round nested PCR-SSP step. Each PCR mix contained a second primer pair amplifying a 429 base pair fragment of the human growth hormone gene, as an internal positive control. ${ }^{10}$

\section{Results}

DNA was extractable from all 23 formalin fixed and paraffin wax embedded tissue fragments as well as from all five peripheral blood samples (case 4). Complete HLADRB1, 3, 4, and 5 genotypes were obtained for all 28 DNA samples, using direct PCR-SSP genotyping for 12 samples (including all five peripheral blood samples) and requiring nested PCR-SSP genotyping for 16 samples. Direct PCR-SSP also allowed successful HLA-DQB1 genotyping for all 12 samples for which this technique was applicable. The only exceptions to this were the failure to detect the expected HLA-DRB3 gene within the dysplastic tissue in case 1 and failure to detect the expected HLA-DQB ${ }^{\star} 05$ allele within the reference tissue in case 8 . Table 1 lists the full results. In cases 1,2 , and 5, the same HLA-DRB1 alleles were detected within the suspected contaminant tissue and the corresponding reference tissue. In the remaining seven cases, the HLADRB1 genotypes detected within the putative contaminants were clearly different to those within the corresponding reference tissue. In four of these cases (cases 3, 4, 8, and 9), the putative contaminant and reference tissue shared one HLA-DRB1 allele while the second was different. In case 4, the HLA-DRB1 genotypes of the putative contaminant and the six potential origins for the contaminant were different, while in case 9, the same HLA-DRB1 alleles were detected within the putative contaminant and the second patient.

\section{Discussion}

Specimen mislabelling or contamination can occur at any stage from the clinic or operating theatre to within the histopathology laboratory and may lead to serious diagnostic uncertainty or error. We have previously developed PCR based HLA class II tissue genotyping methods that were derived from routine DNA based tissue typing techniques for solid organ and bone marrow transplantation matching and disease association studies ${ }^{34}$ and are applicable to the relatively degraded DNA extractable from formalin fixed and paraffin wax embedded 
tissue. ${ }^{12}$ We have also developed related PCR based techniques, again applicable to paraffin wax biopsy specimens, for tissue identification and HLA-disease association studies. ${ }^{12}{ }^{13}$ These techniques are particularly useful for the investigation of individual tissue identity as HLA molecules, involved in modulation of the immune response, are encoded by the most polymorphic loci in the human genome, within the major histocompatibility complex on chromosome six. For example, there are currently 184 identified DRB1 alleles and 31 DQB1 alleles. ${ }^{14}$ HLA encoding genes do not seem to undergo somatic mutation during neoplastic transformation. In fact, only one instance of somatic mutation of an HLA allele has been described, in a case of renal cell carcinoma. ${ }^{15}$ Therefore, the fact that many of the tissue fragments undergoing HLA genotyping in this study were neoplastic in nature is not likely to have detracted from the accuracy of the method for determining individual identity. Identification of different HLA alleles for the locus studied within putative contaminant and reference tissues would therefore indicate different individual origins for the tissues and confirm that contamination or mislabelling had occurred. Conversely, identification of the same HLA alleles within two tissue fragments suggests that they are most likely to be derived from the same individual. However, in this situation it is possible that the two fragments may be derived from different individuals possessing the same detected HLA alleles for the loci examined. The chance of this occurrence depends on the frequency of the HLA alleles in the general population. The HLA-DRB1 alleles vary in frequency among Western Caucasians from $0.7 \%$ for HLA-DRB $1{ }^{\star} 10$ to $33 \%$ for HLA-DRB ${ }^{\star} 04$ and $36 \%$ for HLA-DRB $1{ }^{\star} 15,{ }^{4}$ but as an individual possesses two alleles for each HLA locus, the frequency of possession of a combination of two alleles will be significantly less than the frequency of the individual alleles. For example, the frequency of the HLA-DRB ${ }^{\star} 15,15$ homozygote genotype is approximately $13 \%{ }^{4}$ In this situation, study of further HLA loci or polymorphic non-HLA loci may be required for the determination of individual identity.

Polymerase chain reaction based methods are significantly more accurate than serological or restriction fragment length polymorphism based techniques for HLA class II typing ${ }^{16-19}$ and recent developments have allowed the successful application of PCR-SSP genotyping to the often very limited quantities of DNA extractable from paraffin wax biopsy specimens. ${ }^{1}$ The advent of nested PCR-SSP genotyping has improved the sensitivity of the technique further than was possible without two stage $\mathrm{PCR}^{2}$ and this method is particularly useful for the genotyping of tiny gastrointestinal biopsy specimens.

Within the current series, a full HLA-DRB1 genotype was detectable within all 10 cases, together with identification of the presence of the HLA-DRB3, 4, and 5 genes. Where sufficient DNA was available for direct PCRSSP genotyping, DQB1 alleles were also assignable in each case. Due to linkage disequilibrium, HLA-DRB1 alleles are commonly coinherited with certain DQB1 alleles along with the presence of one of the DRB3, 4, or 5 genes. Therefore, detection of the HLA-DRB3, 4, or 5 genes together with, when possible, the DQB1 genotype, acted as an internal control for the DRB1 genotype and increased the confidence with which the overall HLA class II genotype could be assigned. This contrasts with multiplex microsatellite PCR systems which, while of undoubted validity, contain no equivalent intrinsic control mechanisms. ${ }^{20}{ }^{21}$ In cases 1,2 , and 5, detection of the same HLA-DRB1 alleles within the putative contaminant and the reference tissues indicated that there was no positive evidence that contamination or mislabelling had occurred. However, in case 1 , the metastatic carcinoma within the cervical lymph node was subsequently confirmed as adenocarcinoma and together with the endoscopic appearance of the oesophagus, the fragment of dysplastic squamous epithelium was still believed clinically most likely to represent a contaminant. In case 2, almost the entire gastrectomy specimen was subsequently processed for histological examination, involving the preparation of over 80 slides, the last three of which revealed a small focus of signet ring adenocarcinoma. In this case HLA class II genotyping led to confirmation of the diagnosis of malignancy in this patient and therefore helped to exclude the important possibility that the initial malignant gastric biopsy specimen was derived from a second patient. Although HLA class II genotyping could not prove contamination in case 5 , the presence of the dysplastic mucosal fragment on only a single histological section was strongly suggestive of "carry over". Therefore, this patient could be reassured to a degree, although clinical follow up was continued. In the remaining seven cases, contamination was confirmed by the detected presence of at least one different HLA-DRB1 allele between the contaminant and reference tissues, enabling erroneous suspicion of dysplasia or malignany to be avoided in these patients. These patients could therefore be reassured that their investigations had revealed no evidence of malignancy. In case 9, the same HLA-DRB1 alleles $\left(\right.$ HLA-DRB $\left.1^{\star} 01,15\right)$ were detected within the contaminant and the second patient, strongly suggesting that this patient was the origin of the dysplastic tissue fragment. However, none of the additional patients genotyped in case 4 were identified as potential origins for the neoplastic oesophageal tissue. This suggested that in this case contamination was more likely to have occurred within the laboratory rather than the clinic.

In summary, this study illustrates the particular utility of PCR-SSP HLA class II genotyping for the investigation of potentially mislabelled or contaminated gastrointestinal biopsy samples, specimens in which a diagnosis of neoplasia is often made on minimal tissue and with major implications for the patients involved. The nested modification of the method is particularly applicable to the small 
quantities of DNA extractable from tiny formalin fixed and paraffin wax embedded endoscopic biopsy specimens.

This work was supported in part by a grant from the Wessex Cancer Trust. We would like to thank Dr JM Conroy, Dr N Sheron, Dr N Singh, Dr Q van Wyk, and Dr A Quine for submitting cases for investigation and for providing clinical data.

1 Bateman AC, Sage DA, Al-Talib RK, et al. Investigation of specimen mislabelling in paraffin-embedded tissue using a rapid, allele-specific PCR-based HLA class II typing method. Histopathology 1996;28:169-74.

2 Bateman AC, Hemmatpour SK, Theaker JM, et al. Nested polymerase chain reaction-based HLA class II typing for the unique identification of formalin-fixed and paraffinembedded tissue. F Pathol 1997;181:228-34.

3 Howell WM, Evans PR, Devereux SA, et al. Absence of strong HLA-DR/DQ-DP linkage disequilibrium in the British and French Canadian Caucasoid populations. Eur $\mathcal{F}$ British and French Canadian Cau

4 Howell WM, Leung ST, Jones DB, et al. HLA-DRB, -DQA, and $-D Q B$ polymorphism in celiac disease and enteropathy-associated T-cell lymphoma. Common features and additional risk factors for malignancy. Hum Immunol 1995;43:29-37.

5 Kimura A, Sasazuki T. Eleventh International Histocompatibility Workshop reference protocol for the HLA DNAtyping technique. In: Tsuji K, Aizawa M, Sasazuki T, eds. HLA 1991. Vol 1. Oxford: Oxford University Press, 1992:397-439.

6 Olerup O, Zetterquist H. HLA-DR typing by PCR amplification with sequence-specific primers (PCR-SSP) in 2 hours: an alternative to serological DR typing in clinical practice includin practice including donor-recipient matching in cadaveric transplantation. Tissue Antigens 1992;39:225-35.

7 Olerup O, Zetterquist H. DR "low resolution" PCR-SSP typing - a correction and an update. Tissue Antigen $1993 ; 40: 55-6$.

8 Bunce M, Taylor CJ, Welsh KI. Rapid HLA-DQB typing by eight polymerase chain reaction amplifications with sequence specific primers (PCR-SSP). Hum Immunol 1993; 37:201-6.
9 Marsh SGE, Bodmer JG. HLA class II region nucleotide sequences, 1995. Tissue Antigens 1995;45:258-80.

10 Aldener-Cannavá A, Olerup O. HLA-DQB1 "low resolution" typing by PCR amplification with sequence specific primers (PCR-SSP). Eur F Immunogenet 1994;21:447-55.

11 Olerup $\mathrm{O}$, Zetterquist $\mathrm{H}$. HLA-DRB ${ }^{\star} 01$ subtyping by allele-specific PCR amplification: a sensitive, specific and rapid technique. Tissue Antigens 1991;37:197-204.

12 Bateman AC, Leung ST, Howell WM, et al. Detection of specimen contamination in routine histopathology by HLA class II typing using the polymerase chain reaction and sequence specific oligonucleotide probing. F Pathol 1994; 173:243-8.

13 Bateman AC, Turner SJ, Theaker JM, et al. HLADQB $1{ }^{\star} 0303$ and ${ }^{\star} 0301$ alleles influence susceptibility to and prognosis in cutaneous malignant melanoma in the British caucasian population. Tissue Antigens 1998;52:6773.

14 Bodmer JG, Marsh SGE, Albert ED, et al. Nomenclature for factors of the HLA system, 1996. Eur f Immunogenet 1997; 24:105-51.

15 Brandle D, Brasseur F, Weynants P, et al. A mutated HLA-A2 molecule recognized by autologous cytotoxic $\mathrm{T}$ lymphocytes on a human renal cell carcinoma. 7 Exp Med 1996;183:2501-8.

16 Tiercy JM, Goumez C, Mach B, et al. Application of DR oligotyping to 110 kidney transplant patients with doubtful serological typing. Transplantation 1991;51:1110-14.

17 Middleton D, Savage DA, Cullen C, et al. Discrepancies in serological tissue typing revealed by DNA techniques. Transplant Int 1988;1:161-4.

18 Mytilineos J, Scherer S, Dunkley H, et al. DNA HLA-DR typing of 4000 kidney transplants. Transplantation 1993;55: $782-5$.

19 Howell WM, Evans PR, Wilson PJ, et al. Comparison of serological, cellular, and DNA-RFLP HLA matching in the selection of related bone marrow donors. Bone Marrow Transplant 1989;4:63-8.

20 Jeffreys AJ, Wilson V, Thein SL. Individual-specific "fingerprints" of human DNA. Nature 1985;316:76-9.

21 Lane SA, Taylor GR, Ozols B, et al. Diagnosis of complete molar pregnancy by microsatellites in archival material. $\mathcal{F}$ Clin Pathol 1993;46:346-8. 